\title{
Reflections of science as a product
}

\author{
Judah L. Rosner
}

A new form of title for scientific reports, one which confidently asserts a conclusion rather than implying it, is becoming more prevalent. That's a bad thing.

THE success of molecular biology has led to the expectation that it will continue to deliver answers to basic questions and to provide medical and commercial products. The status and rewards that can nowadays be won by molecular biologists also mean they have to convince journals and funding agencies that their particular line of work is important, even definitive. Have the stakes involved influenced scientists to compromise on their traditional values?

One indication that they have is to be found in the trend of using a particular type of title in scientific reports. The new form is the assertive sentence title (AST). ASTs differ from traditional titles in that they are not clauses but complete sentences that assert a conclusion. "DNA is the Genetic Material" is an AST; "DNA as the Genetic Material" and "DNA, the Genetic Material" are not, nor are questions. The AST has the form of an eternal truth, whereas the traditional choice of words does not state (although it may imply) a conclusion.

Out of a total of 160 titles reprinted in several 'classic papers' for biology ${ }^{\mathrm{t}}$ (spanning the years from 1514 to 1947), genetics $^{2}$ (1865-1955) and molecular genetics $^{3}$ (1913-1963), not one was an assertion. But during the 1970 s and 1980 s the use of ASTs in biological journals has spread like an infectious agent.

\section{Frequencies}

The frequencies of ASTs were measured for biological sciences in samples from PNAS (Proceedings of the National Academy of Sciences, U.S.A.). Between 1960 and 1969 there were no ASTs out of a total of 2,582 titles examined. They first appeared in 1970 (3/429 titles or $0.7 \%)$, and by 1989 the frequency had risen to about $34 \%$. The proportion of ASTs in Cell, a journal of molecular biology launched in 1974, paralleled that in PNAS until 1979 . It increased sharply to $24 \%$ in 1980 and has been around $45 \%$ since 1986 , the highest proportion for any journal examined.

In the figure, AST frequencies in 1982 and 1989 are compared for several other publications. Whereas such titles are infrequent in two chemistry journals, they are common in journals reporting on molecular biology. Given the wide variation from journal to journal, it would seem that editors have a large part in determining AST usage. But the figure shows that molecular and cellular biologists tend to use ASTs much more often than do chemists; I found but one AST out of $378(0.3 \%)$ articles categorized as 'chemistry' in PNAS from 1970 to 1979 , compared to $100 / 3044(3.2 \%)$ for 'biochemistry' over the same period. Furthermore

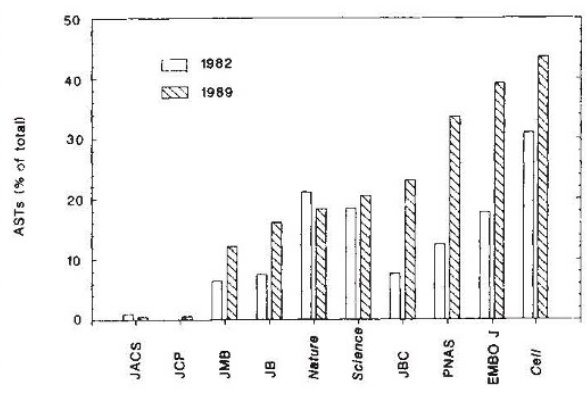

Frequencies of ASTs in ten journals in 1982 and in 1989. Several issues of the journal from a particular year were selected at random and then all of the titles listed in the contents were examined. Each sample consisted of 100 to 300 primary research articles. (JACS, Journal of the American Chemical Society; JCP, Journal of Chemical Physics; JMB, Journal of Molecular Biology; JB, Journal of Bacteriology; JBC, Journal of Biological Chemistry.)

ASTs in Science and Nature are largely in the biological sciences.

What led to the rise in use of these titles in the 1970s, and why are they mainly in molecular biology? I suspect that the answer to both questions lies in the publication in 1965 of James Watson's influential textbook, The Molecular Biology of the Gene $e^{4}$. Watson was an innovator in the use of ASTs as chapter titles and subheadings to stress that biology had come of age - no longer was there a need to beat about the bush; students could be told what is and what isn't known about molecular biology. Indeed, the heading of Chapter 2, "Cells Obey the Laws of Chemistry", boldly states the singular triumph of molecular biology, and lays to rest the bothersome, vitalistic notion that the rules for biology are different from those of chemistry and physics.

Watson's use of ASTs may have encouraged some scientists to adopt them, but why do they continue to rise in popularity? The answer, I believe, lies in the increasing pressure for science to be goalorientated. On this view, the only acceptable outcome of a project is an 'answer', a product. And ASTs provide not only the product but the advertisement for it they help the author to convince the journal, the reader and the granting agency that the article is worth reading and that the work reached a successful conclusion. By advertising the paper in this way, the user of ASTs gains an advantage over the more traditional competitor who may be perceived as someone who looked but could not find. This, in turn, gives rise to 'title wars': a prospective author feels pressure to use ASTs just to keep up with the competition.

\section{Merits}

The merits of the traditional title are these - it states what the work is about in an open-ended tone, inviting the reader to judge the paper by its content not its title, and it reflects the author's appreciation of the possibility of error. In contrast, ASTs are improper and imprudent.

First, they are often patently unprovable (for example declarations that a process requires or does not require a certain component); only when all possible conditions have been tried can that be substantiated. Second, when a title turns out to be in error, the literature is left with that legacy. Even citing the 'good' parts of such a paper will be problematic, because (in many journals) the AST has to appear in the reference citation and valuable work may be buried under an erroneous title. Third, in some cases the AST boldly states a conclusion that is then stated more tentatively in the summary or elsewhere. Such practices misinform the reader who has come to rely on the title for the takehome message. Finally, ASTs trivialize a scientific report by reducing it to a oneliner. There is more to a paper than a single conclusion; the materials, the methods or the data may ultimately prove to be of more value than the advertised conclusion.

It is because ASTs declare science to be a product that they are to be deplored. By adhering to the idea of science as process - not product - we risk less and may ultimately achieve more.

Judah L. Rosner is in the Laboratory of Molecular Biology, National Institute of Diabetes, and Digestive and Kidney Diseases, National Institutes of Health, Bethesda, Maryland 20892, USA.

1. Gabriel, M. L. \& Fogel, S. Great Experiments in Biology (Prentice-Hall, Englewood Cliffs, 1955)

2. Peters, J. A. Classic Papers in Genetics (Prentice-Hall, Englewood Cliffs, 1955).

3. Taylor, J. H. Selected Papers in Molecular Genetics (Academic, New York, 1965).

4. Watson, J. D. The Molecular Biology of the Gene (W. A. Benjamin, Menlo Park, 1965). 